\title{
Emotional Dynamics in the Development of Early Adolescent Psychopathology: A One-Year Longitudinal Study
}

\author{
Anna Neumann • Pol A. C. van Lier • Tom Frijns • \\ Wim Meeus • Hans M. Koot
}

Published online: 15 April 2011

(C) The Author(s) 2011. This article is published with open access at Springerlink.com

\begin{abstract}
This study examined the role of the level and variability of happiness, anger, anxiety, and sadness in the development of adolescent-reported anxiety disorder symptoms, depressive symptoms, and aggressive behavior in 452 adolescents (250 male) followed from age 13 to 14 . Level and between-day variability of emotions were assessed through adolescent report at 3-month intervals across a 1 year period. Level and variability of the four emotions contributed to changes in anxiety disorder and depressive
\end{abstract}

Data of the RADAR study were used. RADAR has been financially supported by main grants from the Netherlands Organisation for Scientific Research (GB-MAGW 480-03-005, GB-MAGW 480-08006), and Stichting Achmea Slachtoffer en Samenleving (SASS), and various other grants from the Netherlands Organisation for Scientific Research, the VU University Amsterdam and Utrecht University.

A. Neumann · P. A. C. van Lier · H. M. Koot

Department of Developmental Psychology, VU University, Amsterdam, the Netherlands

P. A. C. van Lier

e-mail: pac.van.lier@psy.vu.nl

H. M. Koot

e-mail: jm.koot@psy.vu.nl

\author{
A. Neumann $(\bowtie)$ \\ Department of Developmental Psychology, \\ University of Wuppertal, \\ Gaußstrasse 20, \\ 42119 Wuppertal, Germany \\ e-mail: neumann@uni-wuppertal.de \\ T. Frijns $\cdot$ W. Meeus \\ Research Centre Adolescent Development, Utrecht University, \\ Utrecht, the Netherlands \\ T. Frijns \\ e-mail: t.frijns@uu.nl \\ W. Meeus \\ e-mail: w.meeus@uu.nl
}

symptoms more consistently than to changes in aggressive behavior. All four emotions were predictive of changes in internalizing problems, while anger played the most prominent role in the development of aggressive behavior. Variability of emotions contributed to changes in anxiety disorder symptoms, while heightened levels of negative emotions and diminished happiness contributed to changes in depression. Results suggested somewhat stronger effects of negative affect on aggressive behavior for females than for males. Results underscore the role of emotion dysregulation in the development of psychopathology.

Keywords Emotion dysregulation - Emotional dynamics . Internalizing problems $\cdot$ Externalizing problems .

Adolescence

\section{Introduction}

Dysregulated emotions are thought to play a significant role in the etiology and maintenance of many forms of psychopathology (e.g., Bradley 2000; Cole and Hall 2008). In fact, negative or dysregulated emotions are diagnostic symptoms of many disorders as described in the DSM-IV (American Psychiatric Association [APA], 1994). For instance, excessive anxiety and worry, and/or intense fear are implicated in the anxiety disorders, while irritability, anger, and mood lability are implicated in the disruptive behavior disorders (conduct disorder and oppositional defiant disorder; APA, 1994). High levels and prolonged duration of negative emotions and heightened emotional variability may be signs of emotional dysregulation (Cole and Hall 2008). Individual differences in emotion regulation and their relation with the development of psychopathology become especially relevant during the developmental period of adolescence. Adolescence is characterized by an increase in the intensity and frequency of 
(negative) emotions (e.g., Larson and Lampman-Petraidis 1989), heightened levels of emotional variability (Larson et al. 1980), increases in several types of psychopathology (e.g., Moffitt et al. 1996; Wight et al. 2004), and increased demand for independent self-regulation. However, longitudinal studies that address the role of emotion dysregulation in the development of psychopathology in adolescence are scarce. In consequence, little is known about the role of emotion dysregulation in general, and about the role of emotional dynamics (the intensity and variability of emotions; Silk et al. 2003) in the development of adolescent behavioral and emotional problems in particular. The present study focused on the role of the dynamics of four basic emotions (happiness, anger, anxiety, and sadness) in the 1 year change or stability of internalizing and externalizing psychopathology in early adolescence.

Emotional Dynamics and Psychopathology in Adolescence

The term "emotion dysregulation" refers to maladaptive patterns of emotion regulation, that is, to patterns of emotion regulation that are costly in the pursuit of longterm goals such as maintaining social relationships and well-being (Cole and Hall 2008). The dysregulation of emotions may be studied at all different levels of emotion experience, cognition and regulation, such as emotional dynamics (Silk et al. 2003), emotion knowledge (e.g., not knowing that one may experience different emotions at the same time and believing that emotional experiences cannot be modulated; e.g., Meerum-Terwogt and Olthof 1989), difficulties with the use of emotion regulation strategies (e.g., distraction, cognitive reinterpretation; Gross and Thompson 2007), and meta-emotion experiences (e.g., nonacceptance of emotional responses; Gratz and Roemer 2004). We consider emotional experiences and behaviors that are too little, too much, or not appropriate for the situation at hand as signs of dysregulation. This study is focused on emotion dynamics, as these reflect the outcome of regulatory efforts and processes. Specifically, we focus on adolescents' daily experiences of emotions. At the level of emotion dynamics, emotion dysregulation may be reflected in the form of heightened and prolonged negative emotions, and heightened variability of both negative and positive emotions (Cole and Hall 2008; Silk et al. 2003). We define emotional variability (EV) here as "the frequency with which an individual's emotions change, and the extremity of these changes". It should be noted that some variability of emotions, as well as the experience of negative emotions is clearly expected as a result of normal emotional reactivity to environmental stressors. Heightened and prolonged negative emotions and heightened variability, however, may be indications of extreme emotional reactivity and ineffective attempts at emotion regulation.
As indicated previously, early to mid adolescence is an opportune time to study emotional dynamics, and its links with psychopathology. EV was found to be heightened in adolescents compared to both younger children and adults in a study, which assessed EV several times a day over the course of 1 week (Larson et al. 1980). Although heightened EV may partly be a sign of normative developmental changes in adolescence (Larson et al. 1980), comparatively high levels of EV in adolescents may indicate emotional dysregulation, and be indicative of future psychopathology (Schneiders et al. 2006).

Several cross-sectional studies demonstrate that in adolescence, high levels of negative emotions, and high levels of EV are related to symptoms of depression (Larson et al. 1990; Silk et al. 2003), and externalizing problems (Silk et al. 2003). These findings are commonly interpreted as suggesting that emotion dysregulation influences the development of psychopathology. However, given the cross-sectional nature of these studies, the role of emotion dysregulation and EV in the development of psychopathology needs further clarification. Therefore, in the present study, we first tested the hypothesis that high levels of negative emotions, low levels of positive emotions, and high variability of both positive and negative emotions add to the growth or development of different types of psychopathology. In addition, we addressed questions on the importance of intensity versus variability of emotions regarding the development of behavioral/emotional problems, on the specificity of the emotion by psychopathology type association, and the potential gender-specificity of these associations.

A first question is whether it is emotion dysregulation in general or dysregulation of specific emotions that is linked to the development of specific forms of psychopathology. For example, does dysregulated anger relate specifically to aggressive problems, and dysregulated sadness relate specifically to depression? 'Functional continuity' between discrete emotions and specific forms of psychopathology is often assumed (e.g., Cole and Hall 2008; Malatesta and Wilson 1988; Muris and Ollendick 2005). That is, it is assumed that patterns of emotional responding become consolidated over time and consequently lead to specific forms of psychopathology (Zahn-Waxler et al. 2000). Accordingly, individuals who are often fearful are thought to be more likely to develop anxiety-related than other psychological disorders, individuals who are often sad, and experience little happiness will show depressive symptoms, and individuals who are easily angered will display symptoms of disruptive behavior disorders (e.g., Muris and Ollendick 2005). Empirical evidence for specific links between emotions and specific forms of psychopathology in adolescence is rather mixed and may heavily depend on the method used to assess emotions. One cross-sectional study addressing links between observerrated facial expressions of emotions (anger, fear, sadness, and embarrassment) and internalizing and externalizing 
problems in early-adolescent boys, provided support for the idea of specific links (Keltner et al. 1995): boys with externalizing problems showed more anger, and boys with internalizing problems showed more fear. By contrast, studies focusing on the internal experience rather than the expression of emotion tend to find nonspecific associations between discrete emotions and forms of psychopathology. High and variable levels of sadness have been associated with depressive symptoms, and high and variable levels of anger with aggressive problem behavior (Larson et al. 1990; Silk et al. 2003). However, both high and variable anger and anxiety also correlate with depressive symptoms, and high and variable levels of sadness and anxiety correlate with aggressive problem behavior scores in adolescents (Silk et al. 2003). As the present study used adolescent daily reports of experienced emotions we expected to find mainly nonspecific associations between levels and variability of emotions and change in psychopathology.

The study of basic emotional processes in the development and maintenance of psychopathology is not just of theoretical interest, but may also inform preventive intervention and treatment efforts. For instance, the question whether links between discrete negative emotions and psychopathology are better characterized as specific or as general, has potentially important implications for preventive interventions and treatment: if fear, as well as anger and sadness turn out to all play a role in anxiety disorder symptoms, teaching anxious youths how to deal with feelings of fear is insufficient.

Secondly, comparatively little is known about the role of positive emotions in the development of psychopathology. It has long been assumed that elevated negative emotions are a common feature of anxiety and mood disorders, whereas diminished positive affect is specific for mood disorders (e.g., Clark and Watson 1991). A recent review and meta-analysis however, has shown significantly diminished positive affect in social anxiety (Kashdan 2007). Regarding externalizing problems, results of studies that have included indices of positive emotions are mixed: no differences in the display of happiness between aggressive and non-aggressive youth (Orobio de Castro et al. 2005), lower state but not trait happiness in delinquent youth than a comparison group (Plattner et al. 2007), and higher happiness in response to antisocial acts in adolescent males with conduct disorder have all been reported (Cimbora and McIntosh 2003). We hypothesize that, in addition to high levels of negative emotions, low levels of positive emotions are related to increased levels of anxiety, depression, and aggressive behavior.

A third question concerns the gender-specificity of the hypothesized associations. It has been reported that males and females differ in levels of specific types of psychopathology (for a review see Zahn-Waxler et al. 2008), as well as in mean levels and variability of negative emotions (Silk et al. 2003). While it is uncertain whether adolescent sex needs to be taken into account when studying the role of emotional dynamics in the development of internalizing and externalizing problems, it might be that sex-related differences in emotional dynamics in part underlie the established gender differences in levels of internalizing and externalizing problems. For instance, emotion dysregulation may be more strongly associated with internalizing problems for females, and more strongly with aggressive behavior for males. The present study tested whether the associations between emotional dynamics and psychopathology are moderated by adolescent gender.

\section{The Present Study}

The present study addressed the role of the intensity and variability of happiness, anger, anxiety, and sadness in the development of internalizing and externalizing psychopathology in 452 adolescents followed from age 13 to 14 . Adolescents' experiences of emotions were obtained from three 5 day periods, during which adolescents reported on their emotions every day. These intensive assessments of emotional experience contain two strengths: they minimize recall bias, inherent in many other self-report methods of subjective experience, and they maximize ecological validity (Shiffman et al. 2008). We expected that high levels and high variability of negative emotions (anger, anxiety, and sadness), predict the stability of adolescents' anxiety, depression, and aggressive behavior scores from age 13 to age 14, in non-specific ways, thereby extending earlier cross-sectional findings (Silk et al. 2003). Further, we expected that positive emotions play a role in the continuity of symptoms of psychopathology. Specifically, we predicted that low levels of - and high variability in -happiness partly predict the 1 year continuity of adolescents' anxiety, depression, and aggressive behavior scores. An additional question that was tested is whether the level and variability of emotions both contribute uniquely to the development of psychopathology. This is an important question, because the intensity and variability of emotions tend to correlate, but their contributions to symptoms of psychopathology have not been tested in one model (e.g., Silk et al. 2003). Finally, we tested whether the associations between emotional dynamics and psychopathology hold across adolescent gender.

\section{Method}

Participants

The sample used in the present study is the RADAR (Research on Adolescent Development and Relationships) 
study sample (see Van Lier et al. 2011 for an extensive description). Target adolescents were approached in grade 6 at 230 schools in the western and central parts of the Netherlands. A two step inclusion phase (teacher screen, followed by parent interviews) was used to include 497 families (target adolescent, mother, father, and sibling) as well as a friend of the target adolescent in an intensive longitudinal study. In the first step of the inclusion process, adolescents at increased risk for developing externalizing symptoms were oversampled, because of a specific focus of the RADAR study on delinquency development. High risk was determined by teacher ratings of the externalizing scales of the Teacher's Report Form (TRF; Achenbach 1991; Verhulst et al. 1997). Youths who received a T-score $\geq 60$ were labeled as high risk. Teacher information was available on 5,150 children. Due to the full family design and intense data collection, which requires a firm grip of the Dutch language, only adolescents who were identified by teachers as being of Dutch origin were selected $(N=$ 3,237 ). Due to logistical reasons, 1,544 of these children were included in the second sample selection phase. In this phase, youths' parents were approached by phone. Four hundred and sixty-three adolescents were excluded, due to incorrect phone details, or because they did not meet additional requirements for inclusion in the study (both parents present, and presence of a sibling $\geq 10$ years), and 583 actively refused to participate or failed to provide written consent, resulting in the inclusion of 497 families (291 target adolescents at average risk and 206 at high risk for externalizing problems). Family socioeconomic status is low in $11 \%$ of the sample, and the majority of participants (97\%) had a Dutch-Caucasian background. The RADAR study was approved by the medical ethical committee of Utrecht University.

For examining research questions of the present study only adolescent ratings were used. Participants were included if data were available for at least one annual assessments $(N=$ 452, 55.3\% male). Adolescents' mean age at Time 1 was 13.37 years $(S D=0.61)$. Male and female adolescents did not differ regarding risk group status, $\chi^{2}(1)=0.20, p>0.05$, or age, $t(485)=0.65, p>0.05$.

\section{Procedure}

For the present study, data were collected during two home visits (time[T]1 and T5), and three internet assessments (T2, $\mathrm{T} 3$, and T4). Each assessment was separated from the next by a 3 months interval. During the home visits, adolescents completed questionnaires regarding symptoms of anxiety disorders, depression, and aggressive behavior as part of larger questionnaire packages under the supervision of trained interviewers. During the internet assessments, adolescents filled out their experiences of different emotions during that day, via internet. These assessments were completed for 5 consecutive days (Monday to Friday). Each day, participants received an invitation via e-mail to participate (at approximately 5.30 p.m.) and logged in to the RADAR website for data collection. Reminder e-mails were sent to participants who had not completed their assessment $1.5 \mathrm{~h}$ after the initial invitation was sent, followed by text messages and phone calls to those who had still not completed their assessment $1.5 \mathrm{~h}$ after the reminder had been sent.

\section{Measures}

Emotional Dynamics Adolescents completed the Daily Mood Scale, an internet version of the Electronic Mood Device (Hoeksma et al. 2000) at each of the three internet assessments (T2, T3, T4) on 5 consecutive days for a total of 15 days. Each day, adolescents rated the intensity of happiness, anger, anxiety, and sadness on a 9-point Likert scale, ranging from 'not at all' to 'very much' during the daily internet sessions. Each emotion was tapped with three items (glad, happy, and cheerful for happiness; angry, cross, and short-tempered for anger; afraid, anxious, and worried for anxiety; sad, down, and dreary for sadness). These three items per emotion were summed into a total score per day. Cronbach's $\alpha$ s ranged from 0.86 to 0.94 for happiness, from 0.87 to 0.97 for anger, from 0.72 to 0.94 for anxiety, and from 0.92 to 0.97 for sadness across the total 15 days of assessment. Emotion level and variability scores were calculated only for participants who provided valid data on at least 4 days in total or on 3 consecutive days in a week. Due to this conservative way of calculating emotion dynamic scores, emotion data were missing for $7.1 \%$ of the sample at $\mathrm{T} 2$, and for $11.6 \%$ and $18.5 \%$ at $\mathrm{T} 3$ and $\mathrm{T} 4$, respectively.

Levels of Happiness, Anger, Anxiety, and Sadness were calculated for each emotion by summing emotion scores of all days within each week, divided by the number of valid assessments. Next, scores were summed per emotion across the three internet assessments.

Variability of Happiness, Anger, Anxiety, and Sadness The variability scores were calculated per discrete emotion as the difference between scores in adjacent days within each internet assessment week. First, the absolute difference between consecutive data entries was calculated. For each participant, absolute difference scores were summed to a weekly difference score, and divided by the number of valid observations (to control for missing days). The weekly difference scores of the three internet assessment weeks were summed for every emotion, resulting in a single variability score each for happiness, anger, anxiety, and sadness. The validity of this way of calculating EV is supported by a number of findings. First, the measure 
shows moderate intraindividual stability over the course of three $(r \mathrm{~s}=0.35$ and 0.49$)$ and 6 months $(r=0.30)$, supporting the notion that $\mathrm{EV}$ is an individual difference characteristic (Larsen 1987). Second, the notion of EV as a measure of emotional dysregulation receives some support from the fact that correlations between the variability and level of negative emotions are large and positive ( $r \mathrm{~s}=0.51$ to $0.68, p \mathrm{~s}<0.001)$, but large and negative between variability and level of happiness $(r s=$ -0.46 to $-0.51, p \mathrm{~s}<0.001)$. Third, and also supporting the notion of $\mathrm{EV}$ as emotion dysregulation, in a subsample of the present sample $(N=158)$, EV scores were positively related to adolescent self-reported difficulties with emotional clarity and impulse control, as well as with the nonacceptance of emotional responses, low confidence in one's ability to modulate negative emotional responses, and difficulties engaging in goal-directed behavior when distressed ( $r$ s between $0.21, p<0.05$ and $0.34, p<0.001$ ) as assessed with the Difficulties in Emotion Regulation Scale 3 months after the third internet assessment (unpublished data, for the Difficulties in Emotion Regulation Scale see Gratz and Roemer 2004; Neumann et al. 2010). Since the DERS assesses meta-cognitions, not direct experience of emotions, significant but small associations between DERS scales and emotion level and variability are to be expected. Finally, small but significant positive associations have been found between adolescents' and mothers' EV scores (mean $r=0.18, p<0.05$; Neumann et al. 2008).

Anxiety Disorder Symptoms were assessed with the Screen for Child Anxiety Related Emotional Disorders (SCARED; Birmaher et al. 1997). Adolescents' self-reports of scores on the Social Phobia scale (5 items; "I feel shy with people I don't know well") and the Generalized Anxiety scale (8 items; e.g., "I am a worrier") were used. Generalized Anxiety and Social Phobia are especially relevant during adolescence, as indicated by higher standardized mean scores compared to other anxiety disorders across adolescence (Hale et al. 2008). Items are rated on a three-point scale, with 0 (almost never), 1 (sometimes), and 2 (often). The Dutch SCARED has demonstrated good validity in adolescents (Hale et al. 2005), is related in meaningful ways to other measures of childhood anxiety (e.g., RCMAS and FSSC-R; Muris et al. 1998) and demonstrates both sensitivity and specificity in predicting anxiety disorders (Muris et al. 2000). In the present sample Cronbach's $\alpha$ s were 0.77 and 0.80 for Social Phobia and 0.84 and 0.85 for Generalized Anxiety for $\mathrm{T} 1$ and $\mathrm{T} 5$, respectively. The two scales were combined into one 'anxiety' score.

Depressive Symptoms were assessed with the Reynolds Adolescent Depression Scale-2 ${ }^{\text {nd }}$ Edition (RADS-2; Reynolds 2002). Adolescent completed the subscales 'Dysphoric Mood' (8 items; "I feel like crying"), 'Nega- tive Self-Evaluation' (8 items; "I feel I am bad") and 'Somatic Complaints' (7 items; "I am tired"). Items are rated on a four-point scale ranging from 1 (almost never) to 4 (most of the time). The scales were summed into a total depression score. The construct validity of the RADS- 2 has been supported (e.g., scores differentiate between clinically depressed and non-depressed adolescents; Reynolds 2002). Cronbach's alphas of the total depression score were 0.89 at $\mathrm{T} 1$ and 0.94 at $\mathrm{T} 5$ for the present sample.

Aggressive Behavior was assessed through the Youth Self-Report (YSR; Achenbach 1991). Adolescents completed the 19 items of the Aggressive Behavior subscale (e. g., "I physically attack people", and "I argue a lot"). Each item is scored on a three-point scale including 0 (not true), 1 (somewhat or sometimes true), or 2 (very or often true). The validity of the Dutch version of the YSR was demonstrated (Verhulst et al. 1997). In the present sample, Cronbach's alphas were 0.85 at $\mathrm{T} 1$ and 0.88 at T5.

Risk status of the sample was dummy coded $(0=$ low $/$ average risk, $1=$ at-risk). This variable indicates whether or not the adolescent received heightened cut-off scores on teacher and parent-reported externalizing symptoms at the initial screen before entry into this study (age 12). Because it is uncertain how risk status related expected level differences in psychopathology and emotion dynamics may affect their mutual relations, and because this variable represents a nonrandom characteristic of the present sample, we decided to control for Risk Status in our analyses.

\section{Attrition and Missing Data}

Complete data for anxiety disorder and depressive symptoms and aggressive behavior was available for $99 \%$ of the sample at age 13 and $95 \%$ at age 14 . Seventy percent of the sample had complete data for emotion dynamics, while 19\% missed emotion dynamics data for one of the three internet assessments, $7 \%$ missed data on two of the internet assessments, and emotion dynamics data were missing for $4 \%$ of the sample. Adolescents missing emotion dynamics data did not differ from the rest on the sample on any of the psychopathology measures.

\section{Statistical Analyses}

Path analysis models were run in Mplus 5 (Muthén and Muthén 2007). The analyses were performed in four stages. We started off by testing whether the level of the four emotions predicted changes in psychopathology scores from age 13 to 14 . Models were run separately for each form of psychopathology in combination with each emotion. Presence of a significant indirect path from age 13 psychopathology to age 14 psychopathology through the level of an emotion was tested by using the IND command 
in Mplus, which calculates the joined significance of the indirect pathways according to the formula by MacKinnon and colleagues (MacKinnon et al. 2002). Next, we tested whether the Variability of the four emotions predicted the 1 year development of psychopathology, again separately for each form of psychopathology and each emotion. Third, to test whether variability or level is more important in the development of psychopathology, models were run in which the level of an emotion, and its variability were included as possible mediators. All models were controlled for sex and risk status. Finally, to test for sex-differences in associations, multiple group models were run in which the associations were estimated for males and females separately. All models were corrected for non-normal distributions by maximum likelihood estimation with robust standard errors (MLR). Missing data were accounted for by full information maximum likelihood estimation.

\section{Results}

\section{Descriptive Statistics}

Descriptive statistics for all study variables for males and females (dummy coded as 0 and 1 , respectively) are displayed in Table 1. Females reported significantly higher levels of Anxiety Disorder Symptoms and Depressive Symptoms than males at both annual assessments, while no sex differences were found for Aggressive Behavior. Females also reported higher Sadness levels than males, but males and females did not differ on the Levels of Happiness, Anger, and Anxiety. Finally, females showed higher levels of Variability in Happiness and Sadness than males.
Zero-Order Correlations Between All Study Variables

Most zero-order correlations between study variables were statistically significant in the expected direction (Table 2). A few results are highlighted. First, the levels of the three negative emotions (happiness, anger, and anxiety) showed small-to-medium sized positive correlations with the three problem areas, while Happiness Level correlated negatively with the three problem areas. Second, the variability of the three negative emotions and happiness showed small-tomedium positive correlations with Anxiety Disorder Symptoms, Depressive Symptoms, and Aggressive Behavior. Third, the level of the negative emotions showed large positive association with variability of the same emotions, while the level of happiness correlated negatively with Happiness Variability. Finally, anger, anxiety, and sadness level all correlated strongly and negatively with each other, but strongly and positively with happiness level. The variabilities of all four emotions showed strong positive associations.

The Role of Emotion Level in the Development of Psychopathology

Possible effects of the levels of the four emotions in the development of psychopathology were investigated with the models described above. These models were fully saturated (i.e., they have zero degrees of freedom); therefore fit indices cannot be presented. Results are in Table 3. Anxiety Disorder and Depressive Symptoms showed medium stability from age 13 to $14(\beta \mathrm{s}=0.47$ to 0.53 for Anxiety Disorder, and $\beta \mathrm{s}=0.38-0.44$ for Depressive Symptoms), while the stability of Aggressive Behavior was high $(\beta \mathrm{s}=0.58-0.62)$. Anxiety Disorder Symptoms
Table 1 Means and standard deviations of anxiety, depression, aggression, and emotional variability for males and females

T1 Annual home assessment age 13. T5 Annual home assessment age $14 . * p<0.05$. $* * p<0.01$. $* * * \quad p<0.001$.

\begin{tabular}{|c|c|c|c|c|c|c|c|}
\hline & \multicolumn{3}{|l|}{ Male } & \multicolumn{3}{|l|}{ Female } & \multirow[b]{2}{*}{$F$} \\
\hline & Range & M & SD & Range & M & $\mathrm{SD}$ & \\
\hline Anxiety Disorder Symptoms T1 & $13-34$ & 18.15 & 4.17 & $13-38$ & 19.97 & 5.17 & $17.04 * * *$ \\
\hline Anxiety Disorder Symptoms T5 & $13-29$ & 16.53 & 3.82 & $13-38$ & 19.23 & 5.49 & $35.93 * * *$ \\
\hline Depressive Symptoms T1 & $23-71$ & 35.30 & 10.41 & $23-85$ & 41.69 & 11.94 & $10.55^{* *}$ \\
\hline Depressive Symptoms T5 & $23-68$ & 32.07 & 9.26 & $23-80$ & 37.75 & 12.83 & $28.24 * * *$ \\
\hline Aggressive Behavior T1 & $0-24$ & 7.57 & 5.23 & $0-24$ & 7.11 & 4.76 & 0.90 \\
\hline Aggressive Behavior T5 & $0-27$ & 6.41 & 5.67 & $0-26$ & 6.62 & 5.53 & 0.16 \\
\hline Happiness Level & $39-81$ & 65.51 & 10.13 & $21-86$ & 64.44 & 11.53 & 0.81 \\
\hline Happiness Variability & $0-26$ & 8.17 & 5.13 & $0-37$ & 9.69 & 6.61 & $5.53^{*}$ \\
\hline Anger Level & $0-50$ & 19.23 & 9.49 & $9-69$ & 18.50 & 9.92 & 0.46 \\
\hline Anger Variability & $0-38$ & 7.65 & 6.13 & $0-25$ & 7.35 & 5.83 & 0.48 \\
\hline Anxiety Level & $0-46$ & 16.99 & 8.52 & $9-76$ & 18.90 & 10.94 & 3.20 \\
\hline Anxiety Variability & $0-21$ & 5.39 & 4.71 & $0-29$ & 6.53 & 5.60 & 3.86 \\
\hline Sadness Level & $8-40$ & 14.25 & 7.21 & $8-69$ & 16.03 & 9.86 & $3.64 *$ \\
\hline Sadness Variability & $0-29$ & 4.86 & 5.17 & $0-34$ & 6.67 & 6.88 & $6.52 *$ \\
\hline
\end{tabular}


Table 2 Bivariate correlations between study variables

\begin{tabular}{|c|c|c|c|c|c|c|c|c|c|c|c|c|c|c|}
\hline & & 1 & 2 & 3 & 4 & 5 & 6 & 7 & 8 & 9 & 10 & 11 & 12 & 13 \\
\hline 1 & $\begin{array}{l}\text { Anxiety Disorder } \\
\text { Symptoms T1 }\end{array}$ & - & & & & & & & & & & & & \\
\hline 2 & $\begin{array}{l}\text { Anxiety Disorder } \\
\text { Symptoms T5 }\end{array}$ & 0.58 & - & & & & & & & & & & & \\
\hline 3 & Depression T1 & 0.67 & 0.48 & - & & & & & & & & & & \\
\hline 4 & Depression T5 & 0.47 & 0.69 & 0.57 & - & & & & & & & & & \\
\hline 5 & Aggressive Behavior T1 & 0.33 & 0.21 & 0.48 & 0.34 & - & & & & & & & & \\
\hline 6 & Aggressive Behavior T5 & 0.23 & 0.25 & 0.35 & 0.40 & 0.67 & - & & & & & & & \\
\hline 7 & Happiness Level & -0.38 & -0.36 & -0.42 & -0.45 & -0.32 & -0.30 & - & & & & & & \\
\hline 8 & Happiness Variability & 0.27 & 0.36 & 0.24 & 0.33 & 0.22 & 0.18 & -0.58 & - & & & & & \\
\hline 9 & Anger Level & 0.28 & 0.21 & 0.42 & 0.38 & 0.42 & 0.43 & -0.70 & 0.31 & - & & & & \\
\hline 10 & Anger Variability & 0.26 & 0.27 & 0.29 & 0.30 & 0.33 & 0.34 & -0.43 & 0.57 & 0.59 & - & & & \\
\hline 11 & Anxiety Level & 0.42 & 0.41 & 0.50 & 0.50 & 0.36 & 0.38 & -0.68 & 0.38 & 0.78 & 0.41 & - & & \\
\hline 12 & Anxiety Variability & 0.27 & 0.34 & 0.34 & 0.34 & 0.30 & 0.34 & -0.47 & 0.55 & 0.47 & 0.59 & 0.63 & - & \\
\hline 13 & Sadness Level & 0.38 & 0.34 & 0.49 & 0.48 & 0.35 & 0.37 & -0.71 & 0.42 & 0.83 & 0.46 & 0.90 & 0.58 & - \\
\hline 14 & Sadness Variability & 0.32 & 0.36 & 0.34 & 0.40 & 0.30 & 0.28 & -0.51 & 0.71 & 0.48 & 0.64 & 0.53 & 0.72 & 0.64 \\
\hline
\end{tabular}

All correlations are significant with $p$ at least $<0.05$ (one-tailed). T1 Annual home assessment at age 13. T5 Annual home assessment at age 14 .

significantly predicted levels of all four emotions (negatively for happiness), and levels of three emotions (with anger as the exception) predicted later Anxiety Disorder Symptoms beyond the prediction from earlier Anxiety symptoms. Tests of indirect effects showed significant effects of happiness level and high anxiety and sadness in the development of Anxiety Disorders Symptoms from T1 to T5. For Depressive Symptoms, results were highly similar, with the exception that Anger also played a role in the 1 year development of Depressive Symptoms. The development of Aggressive Behavior was predicted by levels of anger, anxiety, and sadness, but not happiness.

The Role of Emotional Variability in the Development of Psychopathology

Results of the models testing for the role of variability of the four emotions in the development of Anxiety Disorder, Depressive, and Aggressive Behavior symptoms, were highly similar to the results for the Levels of the four

Table 3 The role of the levels of discrete emotions in the 1 year development of anxiety, depression, and aggressive behavior

\begin{tabular}{|c|c|c|c|c|c|c|c|c|c|c|c|c|c|}
\hline \multirow[b]{3}{*}{ PP } & \multirow[b]{3}{*}{ Emotion } & & & & \multicolumn{6}{|c|}{ Direct effects } & \multirow{2}{*}{\multicolumn{3}{|c|}{$\frac{\text { Indirect effect }}{\mathrm{PP} \mathrm{T1} \rightarrow \mathrm{EL} \rightarrow \mathrm{PP} \text { T5 }}$}} \\
\hline & & \multicolumn{3}{|c|}{$\mathrm{PP} \mathrm{T} 1 \rightarrow \mathrm{PP}$ T5 } & \multicolumn{3}{|c|}{$\mathrm{PP} \mathrm{T} 1 \rightarrow \mathrm{EL}$} & \multicolumn{3}{|c|}{$\mathrm{EL} \rightarrow \mathrm{PP}$ T5 } & & & \\
\hline & & $\mathrm{B}$ & SE & $\beta$ & B & SE & $\beta$ & B & SE & $\beta$ & $\mathrm{B}$ & SE & $\beta$ \\
\hline \multirow[t]{4}{*}{ Anxiety T5 } & Happy & 0.50 & 0.05 & $0.49 * *$ & -0.29 & 0.04 & $-0.38 * *$ & -0.23 & 0.06 & $-0.17 * *$ & 0.07 & 0.02 & $0.06^{* *}$ \\
\hline & Angry & 0.55 & 0.05 & $0.53 * *$ & 0.20 & 0.04 & $0.30^{* *}$ & 0.10 & 0.07 & 0.07 & 0.02 & 0.01 & 0.02 \\
\hline & Anxious & 0.48 & 0.05 & $0.47 * *$ & 0.28 & 0.04 & $0.41 * *$ & 0.30 & 0.08 & $0.20^{* *}$ & 0.08 & 0.02 & $0.08^{* *}$ \\
\hline & $\mathrm{Sad}$ & 0.52 & 0.05 & $0.51 * *$ & 0.22 & 0.04 & $0.36^{* *}$ & 0.21 & 0.09 & $0.12 *$ & 0.05 & 0.02 & $0.05^{*}$ \\
\hline \multirow[t]{4}{*}{ Depression T5 } & Happy & 0.42 & 0.06 & $0.42 * *$ & -0.13 & 0.02 & $-0.41 * *$ & -0.84 & 0.16 & $-0.27 * *$ & 0.11 & 0.02 & $0.11 * *$ \\
\hline & Angry & 0.44 & 0.06 & $0.44 * *$ & 0.12 & 0.02 & $0.42 * *$ & 0.72 & 0.20 & $0.21^{* *}$ & 0.09 & 0.02 & $0.09 * *$ \\
\hline & Anxious & 0.38 & 0.06 & $0.38 * *$ & 0.14 & 0.02 & $0.49^{* *}$ & 1.03 & 0.21 & $0.29 * *$ & 0.14 & 0.03 & $0.14 * *$ \\
\hline & $\mathrm{Sad}$ & 0.41 & 0.06 & $0.40 * *$ & 0.12 & 0.02 & $0.46^{* *}$ & 1.05 & 0.25 & $0.27 * *$ & 0.12 & 0.03 & $0.12 * *$ \\
\hline \multirow[t]{4}{*}{ Aggressive Beh T5 } & Happy & 0.68 & 0.05 & $0.62 * * *$ & -0.21 & 0.04 & $-0.30^{* *}$ & -0.14 & 0.07 & -0.09 & 0.03 & 0.02 & 0.03 \\
\hline & Angry & 0.64 & 0.05 & $0.58 * * *$ & 0.24 & 0.04 & $0.37 * *$ & 0.29 & 0.10 & $0.17 * *$ & 0.07 & 0.03 & $0.06^{* * *}$ \\
\hline & Anxious & 0.66 & 0.05 & $0.59 * * *$ & 0.21 & 0.04 & $0.34 * *$ & 0.27 & 0.08 & $0.16^{* *}$ & 0.06 & 0.02 & $0.05 * * *$ \\
\hline & Sad & 0.68 & 0.05 & $0.60 * * *$ & 0.18 & 0.03 & $0.31 * *$ & 0.25 & 0.10 & $0.13^{*}$ & 0.05 & 0.02 & $0.04 *$ \\
\hline
\end{tabular}

PP Psychopathology. T1 Annual home assessment age 13. T5 Annual home assessment age 14. EL Emotion Level. Beh Behavior. * $p<0.05$. $* * p<.01 . * * * p<0.001$. 
emotions (see Table 4). The variabilities of all four emotions contributed significantly to the 1 year development of Anxiety Disorder Symptoms and Depressive Symptoms. The variabilities of anger and anxiety also played a role in the 1 year development of Aggressive Behavior, while the variabilities of happiness and sadness did not.

Variability and Levels of Discrete Emotions in the Development of Psychopathology

To test whether emotional level or variability played a bigger role in the development of internalizing problems, models were run in which both the level and the variability of the four discrete emotions were included (see Table 5). Because neither the level nor the variability of happiness played a role in the development of Aggressive Behavior, models for Aggressive Behavior were limited to the three negative emotions. Both Anxiety Disorder Symptoms and Depressive Symptoms showed significant positive links to variability of all emotions, and to anger, anxiety, and sadness levels. Further, Anxiety Disorder and Depressive Symptoms showed significant negative links to happiness level. However, Anxiety Disorder Symptoms were predicted by the variability, but not the levels of the four discrete emotions. Depressive Symptoms on the other hand, were predicted by the levels of happiness (negative association) and anger, anxiety and sadness (positive association), but not by their variability. The indirect effects reflect these findings: the indirect pathways from Anxiety Disorder Symptoms at age 13 to Anxiety Disorder Symptoms at age $14 \mathrm{~T} 1$ via EV (but not emotion levels) were generally statistically significant, as were the indirect pathways from Depression T1 to Depression T5 via emotion levels (but not EV). Significant indirect effects in the development of Aggressive Behavior were found for the levels of anger and sadness, but not anxiety, and not EV. The sizes of the significant indirect effects ranged from 0.01 to 0.14 , indicating small effects.

\section{Gender}

To test for a possible moderating role of gender in associations between emotion dynamics and psychopathology, multigroup models were specified by gender for the final models including level and variability of emotions. A fully constrained model (i.e., a model in which all estimates were constrained to be equal for male and female adolescents) was compared to models in which individual path estimates were freed one at a time (i.e., allowed to vary across gender). Some gender differences were found. Specifically, Anxiety Disorder Symptoms at age 13 predicted Variability in Happiness and Anger more strongly for male $(\beta=0.31$ and $\beta=0.30$, both $p \mathrm{~s}<0.001$ for Happiness and Anger Variability respectively) than for female participants $(\beta=0.21$ and $\beta=0.20$, both $p \mathrm{~s}<0.01$ for Happiness and Anger Variability respectively), $\Delta \chi^{2}(1)=5.40$ for Happiness and $\Delta \chi^{2}(1)=5.09$, for Anger Variability, both $p s<0.05$. Conversely, Depressive Symptoms predicted Happiness Variability for female $(\beta=0.30, p<0.001)$, but not for male $(\beta=0.09, \mathrm{~ns})$ participants, $\Delta \chi^{2}(1)=4.89, p<0.05$. Anxiety and Sadness Levels predicted age 14 Aggressive Behavior for

Table 4 The role of the variability of discrete emotions in the 1 year development of anxiety, depression, and aggressive behavior

\begin{tabular}{|c|c|c|c|c|c|c|c|c|c|c|c|c|c|}
\hline \multirow[b]{3}{*}{ PP } & \multirow[b]{3}{*}{ Emotion } & & & & \multicolumn{6}{|c|}{ Direct effects } & \multirow{2}{*}{\multicolumn{3}{|c|}{$\frac{\text { Indirect effect }}{\mathrm{PP} \mathrm{T} 1 \rightarrow \mathrm{EV} \rightarrow \mathrm{PP} \text { T5 }}$}} \\
\hline & & \multicolumn{3}{|c|}{$\mathrm{PP} \mathrm{T} 1 \rightarrow \mathrm{PP}$ T5 } & \multicolumn{3}{|c|}{$\mathrm{PP} \mathrm{T} 1 \rightarrow \mathrm{EV}$} & \multicolumn{3}{|c|}{$\mathrm{EV} \rightarrow \mathrm{PP} \mathrm{T} 5$} & & & \\
\hline & & $\mathrm{B}$ & SE & $\beta$ & $\mathrm{B}$ & SE & $\beta$ & B & SE & $\beta$ & B & SE & $\beta$ \\
\hline \multirow[t]{4}{*}{ Anxiety T5 } & Happy & 0.51 & 0.05 & $0.50 * * *$ & 0.30 & 0.06 & $0.24 * * *$ & 0.17 & 0.04 & $0.20 * * *$ & 0.05 & 0.02 & $0.05^{* *}$ \\
\hline & Angry & 0.53 & 0.05 & $0.51 * * *$ & 0.33 & 0.06 & $0.26 * * *$ & 0.11 & 0.04 & $0.14 * *$ & 0.04 & 0.02 & $0.04 *$ \\
\hline & Anxious & 0.52 & 0.05 & $0.50 * * *$ & 0.27 & 0.05 & $0.25 * * *$ & 0.18 & 0.05 & $0.19 * * *$ & 0.05 & 0.02 & $0.05^{* *}$ \\
\hline & Sad & 0.51 & 0.05 & $0.50 * * *$ & 0.36 & 0.08 & $0.29 * * *$ & 0.14 & 0.05 & $0.18 * *$ & 0.05 & 0.02 & $0.05^{*}$ \\
\hline \multirow[t]{4}{*}{ Depression T5 } & Happy & 0.49 & 0.05 & $0.48 * * *$ & 0.11 & 0.03 & $0.21 * * *$ & 0.38 & 0.10 & $0.20 * * *$ & 0.04 & 0.02 & $0.04 * *$ \\
\hline & Angry & 0.48 & 0.05 & $0.48 * * *$ & 0.15 & 0.03 & $0.29 * * *$ & 0.31 & 0.11 & $0.17 * *$ & 0.05 & 0.02 & $0.05^{* *}$ \\
\hline & Anxious & 0.48 & 0.05 & $0.48 * * *$ & 0.14 & 0.02 & $0.31 * * *$ & 0.35 & 0.12 & $0.16 * *$ & 0.05 & 0.02 & $0.05^{*}$ \\
\hline & $\mathrm{Sad}$ & 0.47 & 0.05 & $0.46^{* * *}$ & 0.16 & 0.03 & $0.30 * * *$ & 0.40 & 0.11 & $0.21 * * *$ & 0.06 & 0.02 & $0.06^{* *}$ \\
\hline \multirow[t]{4}{*}{ Aggressive Beh T5 } & Happy & 0.71 & 0.05 & $0.64 * * *$ & 0.24 & 0.06 & $0.20 * * *$ & 0.02 & 0.06 & 0.02 & 0.01 & 0.01 & 0.00 \\
\hline & Angry & 0.68 & 0.05 & $0.61 * * *$ & 0.37 & 0.06 & $0.31 * * *$ & 0.10 & 0.04 & $0.11^{*}$ & 0.04 & 0.02 & $0.03 *$ \\
\hline & Anxious & 0.67 & 0.05 & $0.61 * * *$ & 0.27 & 0.05 & $0.27 * * *$ & 0.15 & 0.05 & $0.14 * *$ & 0.04 & 0.01 & $0.04 * *$ \\
\hline & $\mathrm{Sad}$ & 0.69 & 0.05 & $0.63 * * *$ & 0.33 & 0.07 & $0.27 * * *$ & 0.06 & 0.05 & 0.06 & 0.02 & 0.02 & 0.02 \\
\hline
\end{tabular}

PP Psychopathology. T1 Annual home assessment age 13. T5 Annual home assessment age 14. EV Emotional Variability. Beh Behavior. * $p<$ 0.05 . ** $p<0.01$. *** $p<0.001$. 


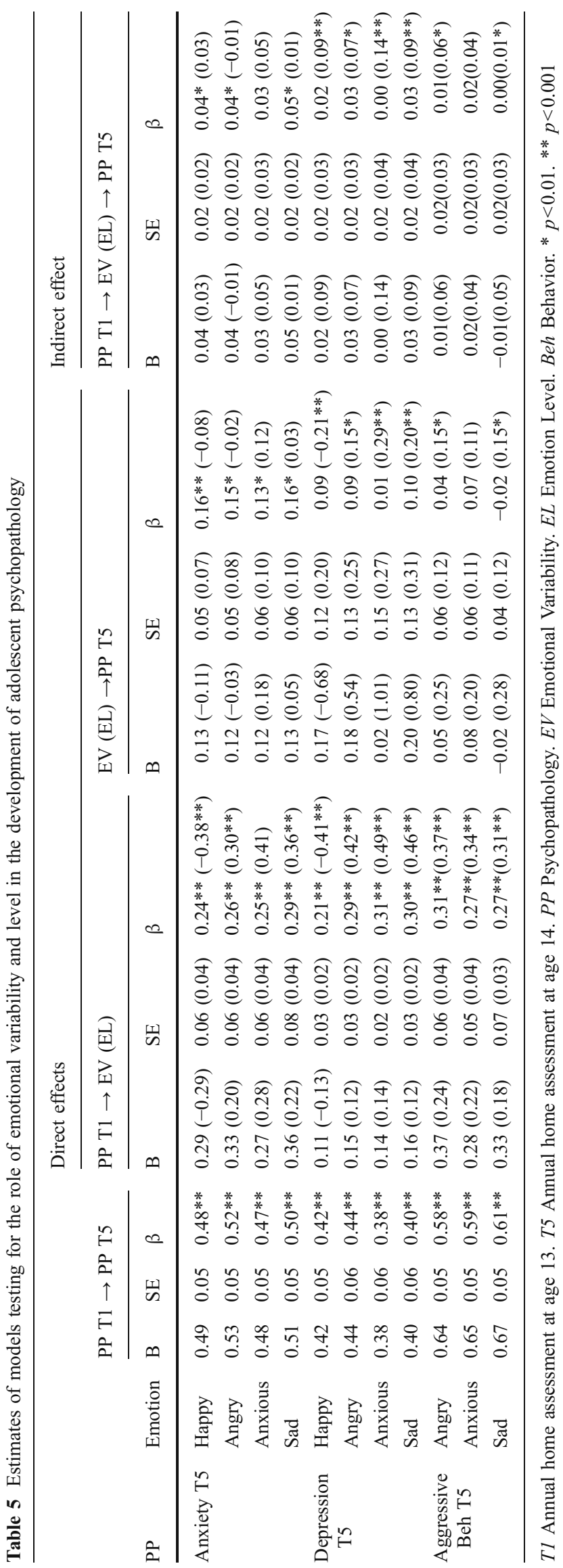

females $(\beta=0.31$ and $\beta=0.23$ for Anxiety and Sadness Levels respectively, both $p \mathrm{~s}<0.01$ ) but not males $\beta=0.06$ and $\beta=0.04$ for Anxiety and Sadness Levels respectively, both $p \mathrm{~s}>0.05, \Delta \chi^{2}(1)=4.34$ for Anxiety Level, and $\Delta \chi^{2}(1)=4.33$ for Sadness Level, both $p \mathbf{s}<0.05$.

\section{Discussion}

The present study examined the role of adolescent emotional dynamics in the development of psychopathology from age 13 to 14 . Results showed that individual differences in the dynamics of happiness, anger, anxiety, and sadness partly account for the development of adolescent anxiety disorder and depressive symptoms, and that the dynamics of the negative emotions, but not happiness, partly account for the development of aggressive behavior. These findings revealed three overarching results. First, most links between the four basic emotions and the three problem areas were non-specific. That is, the dynamics of the four emotions were similarly related to the forms of psychopathology studied. This was particularly true for anxiety disorder and depressive symptoms, while associations between emotion dynamics and aggressive behavior were a bit more specific. In particular, anger played a more consistent role than the other emotions in aggressive behavior. Second, the variability, but not the levels of emotions predicted the development of anxiety disorder symptoms, while the levels, but not the variability, of emotions predicted depressive symptoms and aggressive behavior, when effects of both variability and levels of an emotion were studied in one model. Third, despite the fact that females had higher levels of anxiety and depression, the role of emotional dynamics in the development of psychopathology was similar for both sexes; with the main exception that aggressive behavior was predicted by levels of sadness and anxiety for female, but not for male adolescents.

Building on the assumption that elevated levels of negative emotions, diminished levels of happiness, and elevated emotional variability are all indices of emotion dysregulation, the results add to a growing body of evidence showing that emotion dysregulation predicts symptoms of anxiety, depression and aggressive behavior in children and adolescents (Beauchaine et al. 2007; Bosquet and Egeland 2006; Yap et al. 2008). These findings are consistent with theory proposing that emotion dysregulation underlies the development of psychopathology (Bradley 2000). Our results are also consistent with earlier cross-sectional research linking adolescent negative emotions and emotional variability with symptoms of depression and problem behavior in adolescents (Larson et al. 1990; Silk et al. 2003). 
The finding that the dynamics of happiness, anger, anxiety, and sadness contributed in mostly non-specific ways to different problem areas, may be surprising from the view of functional continuity between emotions and psychopathology. However, our findings are consistent with the notion of a general emotion dysregulation factor (Silk et al. 2003; Steinberg and Avenevoli 2000), and are in line with findings that most forms of psychopathology include dysregulation in more than one discrete emotion. For instance, increased anger (Moscovitch et al. 2008), and diminished happiness (Kashdan 2007) have been implicated in the anxiety disorders, and depression involves the dysregulation of both positive and negative emotions (Gross and Levenson 1997). The fact that, in contrast to the current findings, some studies report specific links between discrete emotions and forms of psychopathology in adolescents (Keltner et al. 1995) and children (e.g., Eisenberg et al. 2001), may be due to differences in the assessment of emotions. While we studied the adolescents' own emotional experience, the above studies used videotaped observations of emotional displays (Keltner et al. 1995) or observer ratings of emotions (Eisenberg et al. 2001). For an emotional experience to be observable, it must have been translated into some form of behavior (e.g., facial or verbal expression). The way in which an internal experience is expressed may very well be an important factor in determining the specific form of psychopathology that one develops. For instance, when both anger and anxiety are often experienced, but the expression of anger is usually suppressed, while anxiety is shown more freely, findings based on observations of these expressions may suggest that the individual is more likely to develop an anxiety disorder than antisocial personality disorder. As our results show, however, at an experiential level, high levels and variability of negative emotions (and low levels of positive emotions), are implicated in non-specific ways in adolescent mental health problems. This suggests that the specificity hypothesis does not hold for experiential data. Future research needs to explicitly address the question of what determines emotional expression - candidate variables may be intrapersonal variables, such as behavioral inhibition versus behavioral activation (Gray 1982) as well as social variables, such as cultural and gender specific display rules for emotions (e.g., Brody 2000).

This non-specificity may have implications for our understanding of comorbidity. Anxiety disorders, mood disorders, and disruptive disorders show strong co-occurrence in childhood and adolescence. Caron and Rutter (1991) suggest that one possible reason for overlap between two disorders is that they share the same risk factor or factors. This possibility arises from the fact that many psychiatric disorders are multifactorial in origin and that many causal factors are not diagnosis-specific. However, it is not known whether the shared risk factors mechanism of several factors mentioned by these authors (e.g., temperamental variables, family adversity) does in fact account for patterns of comorbidity. It may well be that emotional dysregulation is one of the risk mechanisms underlying multiple disorders, including the ones addressed in the present study.

Some specificity in links between the type of emotion dynamics and forms of psychopathology was still found: increased variability in all emotions was specifically related to anxiety disorder symptoms, while elevated levels of negative emotions and diminished levels of happiness, but not their variability were specifically related to depression. This finding is consistent with reports that the development of anxiety and depressive disorder symptoms of adolescents from the general community, though parallel, occurs as two distinct disorders (Hale et al. 2009), and may have implications for our understanding of depression versus anxiety disorder. What differentiates individuals at risk for depression from individuals at risk for anxiety disorders, may be that the former experience "learned helplessness" (Abramson et al. 1978), while the latter alternate between hope and fear. Thus, depression may be characterized by the belief that there is little that one can do about one's situation in general, and about one's affective state in particular. In contrast, inherent in the excessive worry experienced by individuals at risk for anxiety disorders, may be not only the fear that things will not turn the way one wishes, but also the hope that they will-leading to rapid changes in emotional states. This interpretation, though speculative, is consistent with findings that symptoms of anxiety often precede symptoms of depression (Cole et al. 1998). While there is still hope in anxiety, it is lost in depression, as reflected in the present study by heightened variability of emotional states in anxiety, and rather high and stable levels of negative emotions, and low happiness in depression. A recent study of emotional inertia (defined as 'the degree to which emotional states are resistant to change'; Kuppens et al. 2010, p. 984) in 16 year olds, showed that emotional inertia was significantly higher in adolescents who met DSM-IV criteria for current major depressive disorder than in a control group. Taken together with the results of the present study, these findings suggest, that depression in younger adolescents is characterized mainly by high levels of negative emotions, while depression in older adolescents with clinically high symptom levels, is (additionally) characterized by high emotional inertia. Future research may investigate the role of emotional dynamics in the development of anxiety and depression using longer timeframes, comparing clinical and non-clinical groups, and including more cognitive predictors such as hope and helplessness.

While some associations between anxiety and sadness dynamics on the one hand, and the development of 
aggressive behavior on the other hand, were found, anger clearly played the most prominent role in adolescents' aggressive behavior. Compared to the internalizing problems, aggressive behavior thus appears less emotional. This is in line with research showing that self-reported emotion dysregulation (Neumann et al. 2010) explains more of the variance of internalizing than of externalizing problems. However, it is still possible, that certain forms of externalizing problems, which were not studied in the present investigation, are more emotional. It has been suggested that reactive aggression is more emotional than proactive aggression, and it has indeed been found that reactive aggression is related to poorly regulated responses to emotional stimuli (Vitaro et al. 2002), while proactive aggression is related to callousness and emotional shallowness (Frick et al. 2003; Marsee and Frick 2007).

In addition to sex differences in the levels of emotion variability and anxiety and depression, some sex differences in associations between psychopathology and emotion dynamics were found. Anxiety disorder symptoms predicted happiness and anger variability more strongly for males, and sadness and anxiety predicted aggressive behavior for female, but not male adolescents. These gender differences seem to suggest that more emotions are involved in the less gendertypical disorders, akin to the gender paradox as proposed for conduct problems (Tiet et al. 2001). However, breaking this pattern, depressive symptoms predicted happiness variability for females, but not males. The gender differences that we found are thus not entirely conclusive, and it should also be noted, that most paths between emotion dynamics and psychopathology were not moderated by gender. It might be that sex differences in emotion variability and in psychopathology, and their potential associations are not yet distinct enough in early adolescence. Research has shown that sex-differences in (especially internalizing) psychopathology emerge/widen throughout adolescence (Zahn-Waxler et al. 2008). Further research across a larger age range is needed to adequately test effects of gender as a moderating variable in associations between emotion dynamics and psychopathology.

Some limitations of this study need to be mentioned. First, the sample consisted of white, mostly middle-class Dutch adolescents; thus caution should be exercised in generalizing the results to the general adolescent population. It is also unclear how the present results generalize to clinical populations. Further, the sample represented only a small age-range of adolescence. Another limitation is that $\mathrm{EV}$ and internalizing and externalizing problems were assessed by adolescent self-report only. Associations between EV and psychopathology might have been inflated by shared source variance. Subjects who experience high levels of negative emotions and heightened emotional variability, might also report more symptoms of psychopa- thology. The subjective experience of emotions and psychological well being is important; nevertheless, it would be interesting to see if the associations found in the present study hold, if reports by other informants, diagnoses, or observational methods are used. Also, EV is generally assessed over several days, with multiple assessments of emotional intensity per day (e.g., Larson et al. 1980; Silk et al. 2003). In the present study, EV was indexed as EV over the course of three one-week periods, with one assessment of emotional intensity per day. It would be interesting to see if the present results replicate with EV measured intensively over the course of one or several days. However, again, our findings were in accordance with theoretical assumptions and earlier empirical studies. Therefore, not having a within-day variability measure of EV did not seem to overly affect our results.

Clearly, especially with regard to the variability of emotions, more work is needed to understand its nature and development in adolescence, and how it relates to emotional reactivity to stressors, and emotion regulation strategies. For instance, how do emotional reactivity and emotion regulation strategies interact, i.e., can some highly emotionally reactive individuals modulate their emotions in such effective ways that emotion dysregulation does not result? Further, concerning the link to psychopathology, an important issue would be: when does EV become so strong that it becomes a risk for the development of emotional problems? Clearly, emotions and moods that resist change are maladaptive as well (Cole and Hall 2008), and to some extent EV is normative in response to events that are common in adolescence, such as difficulties with peers, or major disappointments in school. Since our measure of EV indexed 3 weeks, separated by 3 months, we are confident that such occurrences have not had a major impact on our results, but it would nevertheless be informative to study $\mathrm{EV}$ in the context of adolescents' daily lives.

Future studies of the sources and consequences of EV may also want to include pubertal status, as pubertal status has been related to both depressive and aggressive affect (Brooks-Gunn et al. 1994), and recent evidence shows that pubertal status and emotional reactivity to experimentally induced stress interact in the prediction of internalizing symptoms (Leen-Feldner et al. 2007).

In sum, results of the present study imply that when studying the emotional underpinnings of (internalizing) psychopathology, researchers may want to focus less on the specific emotions, and more on the general form the dysregulation takes, as indicated by high levels of negative, and low levels of positive emotions, or highly variable emotions. In addition to application to research and theory, the study of basic emotional processes in adolescence is also informative for prevention and intervention efforts, as early forms of emotion dysregulation can indicate risk for 
psychopathology (Cole and Hall 2008). An important message regarding intervention from the present study, then, is to consider the entire emotional spectrum.

Open Access This article is distributed under the terms of the Creative Commons Attribution Noncommercial License which permits any noncommercial use, distribution, and reproduction in any medium, provided the original author(s) and source are credited.

\section{References}

Abramson, L. Y., Seligman, M. E. P., \& Teasdale, J. (1978). Learned helplessness in humans: critique and reformulation. Journal of Abnormal Psychology, 87, 49-74.

Achenbach, T. M. (1991). Manual for the youth self report and 1991 profiles. Burlington: Department of Psychiatry, University of Vermont.

American Psychiatric Association. (1994). Diagnostic and statistical manual of mental disorders (4th ed.). Washington: American Psychiatric Association.

Beauchaine, T. P., Gatzke-Kopp, L., \& Mead, H. K. (2007). Polyvagal theory and developmental psychopathology: emotion dysregulation and conduct problems from preschool to adolescence. Biological Psychology, 74, 174-184.

Birmaher, B., Ketharpal, S., Brent, D., Cully, M., Balach, M., Kaufman, J., et al. (1997). The Screen for Childhood Anxiety Related Emotional Disorders (SCARED): Scale construction and psychometric characteristics. Journal of the American Academy of Child and Adolescent Psychiatry, 36, 545-553.

Bosquet, M., \& Egeland, B. (2006). The development and maintenance of anxiety symptoms from infancy through adolescence in a longitudinal sample. Development and Psychopathology, 18, 517-550.

Bradley, S. J. (2000). Affect regulation and the development of psychopathology. New York: Guilford Press.

Brody, L. R. (2000). The socialization of gender differences in emotional expression: Display rules, infant temperament, and differentiation. In A. H. Fischer (Ed.), Gender and emotion: Social psychological perspectives (pp. 24 47). New York: Cambridge University Press.

Brooks-Gunn, J., Graber, J. A., \& Paikoff, R. L. (1994). Studying links between hormones and negative affect: models and measures. Journal of Research on Adolescence, 4, 469-486.

Caron, C., \& Rutter, M. (1991). Comorbidity in child psychopathology: concepts, issues, and research strategies. Journal of Child Psychology and Psychiatry, 32, 1063-1080.

Cimbora, D. M., \& McIntosh, D. N. (2003). Emotional responses to antisocial acts in adolescent males with conduct disorder: a link to affective morality. Journal of Clinical Child and Adolescent Psychology, 32, 296-301.

Clark, L. A., \& Watson, D. (1991). Tripartite model of anxiety and depression: psychometric evidence and taxonomic implications. Journal of Abnormal Psychology, 100, 316-336.

Cole, P. M., \& Hall, S. E. (2008). Emotion dysregulation as a risk factor for psychopathology. In T. P. Beauchaine \& S. P. Hinshaw (Eds.), Child and adolescent psychopathology (pp. 265-298). Hoboken: Wiley.

Cole, D. A., Peeke, L. G., Martin, J. M., Truglio, R., \& Seroczynski, A. D. (1998). A longitudinal look at the relation between depression and anxiety in children and adolescents. Journal of Consulting and Clinical Psychology, 66, 451-460.

Eisenberg, N., Cumberland, A., Spinrad, T. L., Fabes, R. A., Shepard, S. A., Reiser, M., et al. (2001). The relations of regulation and emotionality to children's externalizing and internalizing problem behavior. Child Development, 72, 1112-1134.
Frick, P. J., Cornell, A. H., Bodin, S. D., Dane, H. E., Barry, C. T., \& Loney, B. R. (2003). Callous-unemotional traits and developmental pathways to severe conduct problems. Developmental Psychology, 39, 246-260.

Gratz, K. L., \& Roemer, L. (2004). Multidimensional assessment of emotion regulation and dysregulation: development, factor structure, and initial validation of the difficulties in emotion regulation scale. Journal of Psychopathology and Behavioral Assessment, 26, 41-54.

Gray, J. A. (1982). The neuropsychology of anxiety. New York: Oxford University Press.

Gross, J. J., \& Levenson, R. W. (1997). Hiding feelings: the acute effects of inhibiting negative and positive emotions. Journal of Abnormal Psychology, 106(1), 95-103.

Gross, J. J., \& Thompson, R. A. (2007). Emotion regulation: Conceptual foundations. In J. J. Gross (Ed.), Handbook of emotion regulation (pp. 3-24). New York: Guilford Press.

Hale, W., Raaijmakers, Q., Muris, P., \& Meeus, W. (2005). Psychometric properties of the screen for child anxiety related emotional disorders (SCARED) in the general adolescent population. Journal of the American Academy of Child and Adolescent Psychiatry, 44, 283-290.

Hale, W., Raaijmakers, Q., Muris, P., Van Hoof, A., \& Meeus, W. (2008). Developmental trajectories of adolescent anxiety disorder symptom factors: A 5 year prospective community study. Journal of the American Academy of Child and Adolescent Psychiatry, 47, 556-564.

Hale, W., Raaijmakers, Q., Muris, P., Van Hoof, A., \& Meeus, W. (2009). One factor or two parallel processes? Comorbidity and development of adolescent anxiety and depressive disorder symptoms. Journal of Child Psychology and Psychiatry, 50, $1218-1226$.

Hoeksma, J. B., Sep, S. M., Vester, F. C., Groot, P. F. C., Sijmons, R., \& deVries, J. (2000). The electronic mood device: design, construction and application. Behavior Research Methods, Instruments \& Computers, 32, 322-326.

Kashdan, T. B. (2007). Social anxiety spectrum and diminished positive experiences: theoretical synthesis and meta-analysis. Clinical Psychology Review, 27, 348-365.

Keltner, D., Moffitt, T. E., \& Stouthamer-Loeber, M. (1995). Facial expressions of emotion and psychopathology in adolescent boys. Journal of Abnormal Psychology, 104, 644-652.

Kuppens, P., Allen, N. B., \& Sheeber, L. B. (2010). Emotional inertia and psychological maladjustment. Psychological Science, 27, 984-991.

Larsen, R. J. (1987). The stability of mood variability: a spectral analytic approach to daily mood assessments. Journal of Personality and Social Psychology, 52, 1195-1204.

Larson, R., Csikszentmihalyi, M., \& Graef, R. (1980). Mood variability and the psychosocial adjustment of adolescents. Journal of Youth and Adolescence, 9, 469-490.

Larson, R., \& Lampman-Petraidis, C. (1989). Daily emotional states as reported by children and adolescents. Child Development, 60 , $1250-1260$.

Larson, R., Raffaelli, M., Richards, M. H., Ham, M., \& Jewell, L. (1990). Ecology of depression in late childhood and early adolescence: a profile of daily states and activities. Journal of Abnormal Psychology, 99, 92-102.

Leen-Feldner, E. W., Reardon, L. E., \& Zvolensky, M. J. (2007). Pubertal status and emotional reactivity to a voluntary hyperventilation challenge predicting panic symptoms and somatic complaints. Behavior Modification, 31, 8-31.

MacKinnon, D. P., Lockwood, C. M., Hoffman, J. M., West, S. G., \& Sheets, V. (2002). A comparison of methods to test mediation and other intervening variable effects. Psychological Methods, 7, 83-104. 
Malatesta, C. Z., \& Wilson, A. (1988). Emotion cognition interaction in personality development: a discrete emotions, functionalist analyses. British Journal of Social Psychology, 27, 91-112.

Marsee, M. A., \& Frick, P. J. (2007). Exploring the cognitive and emotional correlates to proactive and reactive aggression in a sample of detained girls. Journal of Abnormal Child Psychology, 35, 969-981.

Meerum-Terwogt, M., \& Olthof, T. (1989). Awareness and selfregulation of emotion in young children. In C. Saarni \& P. L. Harris (Eds.), Children's understanding of emotion (pp. 209237). Cambridge: Cambridge University Press.

Moffitt, T. E., Caspi, A., Dickson, N., Silva, P., \& Stanton, W. (1996). Childhood-onset versus adolescent-onset antisocial conduct problems in males: natural history from ages 3-18 years. Development and Psychopathology, 8, 399-424.

Moscovitch, D. A., McCabe, R. E., Antony, M. M., Rocca, L., \& Swinson, R. P. (2008). Anger experience and expression across the anxiety disorders. Depression and Anxiety, 25, 107-113.

Muris, P., Merckelbach, H., Mayer, B., van Brakel, A., Thissen, S., Moulaert, V., et al. (1998). The Screen for Child Anxiety Related Emotional Disorders (SCARED) and traditional childhood anxiety measures. Journal of Behavior Therapy and Experimental Psychiatry, 29, 327-339.

Muris, P., Merckelbach, H., Mayer, B., \& Prins, E. (2000). How serious are common childhood fears? Behaviour Research and Therapy, 38, 217-228.

Muris, P., \& Ollendick, T. H. (2005). The role of temperament in the etiology of child psychopathology. Clinical Child and Family Psychology Review, 8, 271-289.

Muthén, L. K., \& Muthén, B. O. (2007). MPlus: statistical analysis with latent variables user's guide (5th ed.). Los Angeles: StatModel.

Neumann, A., Lichtwarck-Aschoff, A., van Lier, P. A. C., Frijns, T., Meeus, W., \& Koot, H. M. (2008, March). Emotionality in the mother-adolescent relationship. In A. Neumann \& A. M. Willemen (Chairs), Family context and adolescent emotion regulation. Symposium conducted at the biennial meeting of the Society for Research on Adolescence, Chicago, IL.

Neumann, A., van Lier, P. A. C., Gratz, K. L., \& Koot, H. M. (2010). Multidimensional assessment of emotion regulation difficulties in adolescents using the difficulties in emotion regulation scale. Assessment, 17, 138-149.

Orobio de Castro, B., Merk, W., Koops, W., Veerman, J. W., \& Bosch, J. D. (2005). Emotions in social information processing and their relations with reactive and proactive aggression in referred aggressive boys. Journal of Clinical Child and Adolescent Psychology, 34, 105-116.

Plattner, B., Karnik, N., Jo, B., Hall, R. E., Schallauer, A., Carrion, V., et al. (2007). State and trait emotions in delinquent adolescents. Child Psychiatry and Human Development, 38, 155-169.
Reynolds, W. M. (2002). Reynolds Adolescent Depression Scale $-2^{\text {nd }}$ Edition: Professional manual. Lutz, FL: Psychological Assessment Resources.

Schneiders, J., Nicolson, N. A., Berkhof, J., Feron, F. J., van Os, J., \& deVries, M. W. (2006). Mood reactivity to daily negative events in early adolescence: relationship to risk for psychopathology. Developmental Psychology, 42, 543-554.

Shiffman, S., Stone, A. A., \& Hufford, M. R. (2008). Ecological momentary assessment. Annual Review of Clinical Psychology, 4, 1-32.

Silk, J. S., Steinberg, L., \& Morris, A. S. (2003). Adolescents' emotion regulation in daily life: links to depressive symptoms and problem behavior. Child Development, 74, 1869-1880.

Steinberg, L., \& Avenevoli, S. (2000). The role of context in the development of psychopathology: a conceptual framework and some speculative propositions. Child Development, 71, 66-74.

Tiet, Q. Q., Wasseman, G. A., Loeber, R., McReynolds, L. S., \& Miller, L. S. (2001). Developmental and sex differences in types of conduct problems. Journal of Child and Family Studies, 10, 181-197.

Van Lier, P. A. C., Frijns, T., Neumann, A., den Exter Blokland, E., Koot, H. M., \& Meeus, W. (2011). The RADAR study: Design, description of sample, and validation of cohort assignment. Manuscript submitted for publication.

Verhulst, F. C., van der Ende, J., \& Koot, H. M. (1997). Handleiding voor de Youth Self-Report (YSR) [Manual for the Youth Self-Report]. Rotterdam, The Netherlands: Afdeling Kinder- en jeugdpsychiatrie, Sophia Kinderziekenhuis/ Academisch Ziekenhuis Rotterdam/Erasmus Universiteit Rotterdam

Vitaro, F., Brendgen, M., \& Tremblay, R. E. (2002). Reactively and proactively aggressive children: antecedent and subsequent characteristics. Journal of Child Psychology and Psychiatry, 43, 495-505.

Wight, R. G., Sepúlveda, J. E., \& Aneshensel, C. S. (2004). Depressive symptoms: how do adolescents compare with adults? Journal of Adolescent Health, 34, 314-323.

Yap, M. B. H., Allen, N. B., \& Ladouceur, C. D. (2008). Maternal socialization of positive affect: the impact of invalidation on adolescent emotion regulation and depressive symptomatology. Child Development, 79, 1415-1431.

Zahn-Waxler, C., Klimes-Dougan, B., \& Slattery, M. J. (2000). Internalizing problems of childhood and adolescence: prospects, pitfalls, and progress in understanding the development of anxiety and depression. Development and Psychopathology, 12, 443-446.

Zahn-Waxler, C., Shirtcliff, E. A., \& Marceau, K. (2008). Disorders of childhood and adolescence: gender and psychopathology. Annual Review of Clinical Psychology, 4, 275-303. 